Textures and Microstructures, Vol. 30, pp. 133-143 Reprints available directly from the publisher Photocopying permitted by license only
(C) 1998 OPA (Overseas Publishers Association) N.V.

Published by license under the Gordon and Breach Science Publishers imprint. Printed in India.

\title{
THE EFFECT OF SOLLER SLIT AND MONOCHROMATOR USED FOR BACKGROUND REDUCTION IN TEXTURE MEASUREMENTS
}

\author{
K. VAN ACKER* and P. VAN HOUTTE \\ Department of Metallurgy and Materials Engineering, Katholieke \\ Universiteit Leuven, de Croylaan 2, 3001 Heverlee, Belgium
}

\author{
(Received 6 September 1996)
}

\begin{abstract}
An X-ray texture goniometer equipped with a vertical Soller slit and a monochromator in the secondary beam in combination with $\mathrm{Cu}$ radiation is found to be useful for pole figure measurements on different materials, e.g. $\mathrm{Al}$ and $\mathrm{Fe}$, as well as for low incident beam angle measurements on thin coatings. The obtained ODF's are less sensitive to errors in the procedure used for background correction of the pole figures than obtained with the classical setup used for pole figure measurements. The reason for that is the drastically ameliorated Intensity/Background (I/B) ratio by the use of the monochromator. Examples for cold rolled $\mathrm{Fe}$ and $\mathrm{Al}$ are presented. One of the merits of the new setup is that pole figures of $\mathrm{Al}, \mathrm{Cu}, \mathrm{Ti}, \mathrm{Fe}$ can all be measured by using $\mathrm{Cu} \mathrm{K} \alpha$ radiation, thus avoiding frequent and time-consuming exchanges of $\mathrm{X}$-ray tubes.
\end{abstract}

Keywords: Texture goniometer; Soller slit; Monochromator; Intensity to background ratio

\section{INTRODUCTION}

An X-ray texture goniometer equipped with a vertical Soller slit and a monochromator in the secondary beam (see Fig. 1) is mainly used for pole figure measurements on thin coatings with low incident beam angles (Bonarski et al., 1994; Moreau et al., 1994; Schubert et al., 1993; Szpunar and Blandford, 1994; Van Acker et al., 1994). However, such an attachment also proves useful for classical pole figure measurements

\footnotetext{
${ }^{*}$ Corresponding author.
} 


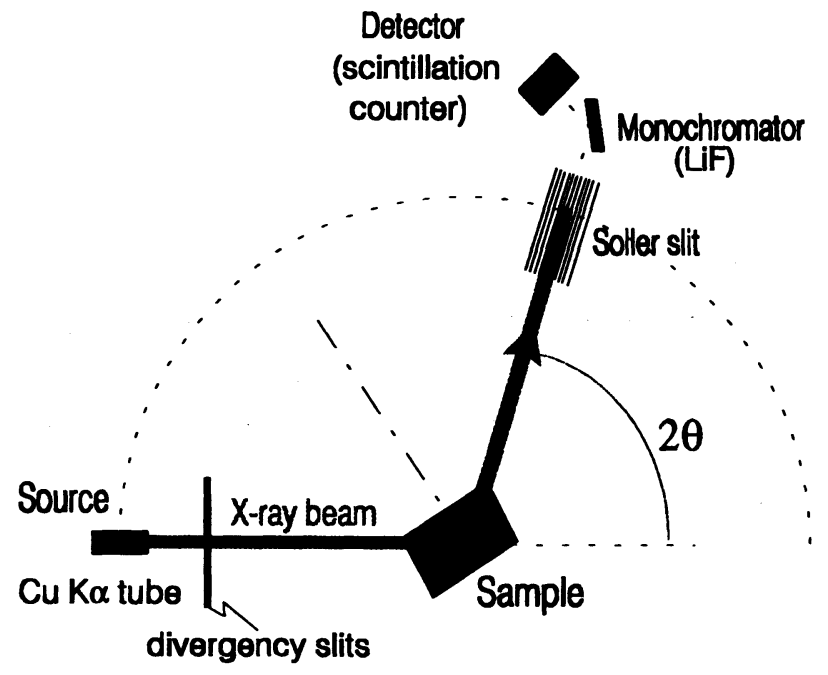

FIGURE 1 Sketch of the presented texture goniometer setup.

because the background intensity is drastically reduced. The setup also allows for pole figure measurements on steel samples using copper radiation. This is not possible in a conventional texture goniometer, because of the strong fluorescence radiation. As a result, it becomes possible to measure pole figures of $\mathrm{Cu}, \mathrm{Al}, \mathrm{Ti}$, and steel samples without changing the $\mathrm{X}$-ray tube, which is very convenient.

In a first part of this paper, some important features of the presented setup are discussed, namely the background reduction and the use of a large divergency slit at the source. Then, texture measurements on cold rolled $\mathrm{Al}$ and on cold rolled $\mathrm{Fe}$ are compared with the classical measurements. It will be seen that the obtained ODF has a higher texture index when the proposed setup is used. It will be shown by a Monte Carlo simulation that the accuracy increases while the Intensity/ Background (I/B) ratio becomes higher.

\section{FEATURES OF THE PRESENTED SETUP}

\subsection{Background Reduction}

The use of a monochromator reduces the background intensity drastically. It is even possible to observe accurately the diffraction peaks of 


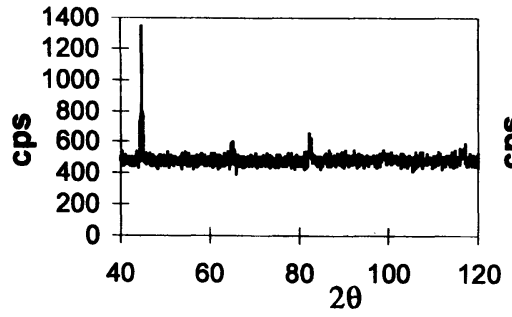

a.

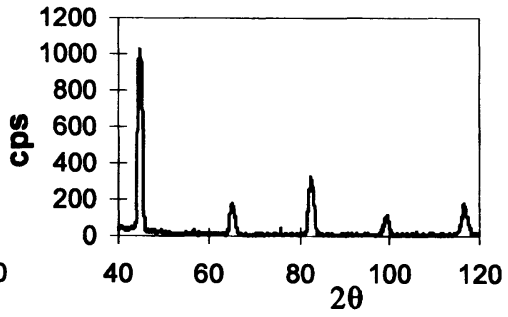

b.

FIGURE 2 Comparison of a diffraction on a Fe powder sample measured with $\mathrm{Cu}$ $\mathrm{K} \alpha$, (a) without monochromator and (b) with monochromator.

TABLE I I/B ratios for Fe powder sample

\begin{tabular}{lcc}
\hline Fe powder & $\begin{array}{l}\text { I/B ratio without } \\
\text { monochromator }\end{array}$ & $\begin{array}{l}\text { I/B ratio with } \\
\text { present setup }\end{array}$ \\
\hline$(110)$ & 2.9 & 40.0 \\
$(200)$ & 2.2 & 31.0 \\
$(211)$ & 2.3 & 34.7 \\
$(310)$ & 2.1 & 30.0 \\
\hline
\end{tabular}

TABLE II I/B ratios for Al powder sample

\begin{tabular}{lcc}
\hline Al powder & $\begin{array}{c}\text { I/B ratio without } \\
\text { monochromator }\end{array}$ & $\begin{array}{c}\text { I/B ratio with } \\
\text { present setup }\end{array}$ \\
\hline$(111)$ & 29.0 & 62.5 \\
$(200)$ & 26.5 & 60.0 \\
$(220)$ & 17.8 & 53.1 \\
$(311)$ & 21.5 & 54.7 \\
\hline
\end{tabular}

Fe with $\mathrm{Cu} \mathrm{K} \alpha$ radiation in the presented setup (see Fig. 2). The I/B ratio increases by a factor 10 or more in the case of Fe (see Table I). The I/B ratio even increases by a factor 2 for $\mathrm{Al}$ (see Table II). Classically, $\mathrm{Cu} \mathrm{K} \alpha$ radiation is considered as well suited for texture measurements on $\mathrm{Al}$. This amelioration of the I/B ratio for $\mathrm{Fe}$ and for other materials is the major advantage of the presented technique.

\subsection{Divergency Slit Angle}

Classically, a small divergency angle at the source is chosen. The detector on the contrary has a large opening in order to measure a range in $2 \theta$-angles, and in order to capture the integrated intensity of the diffraction peak, instead of the intensity at a single $2 \theta$-angle 


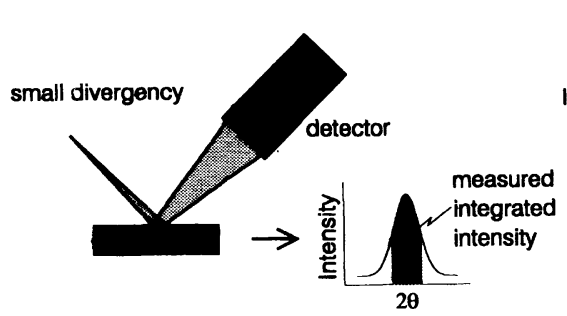

a.

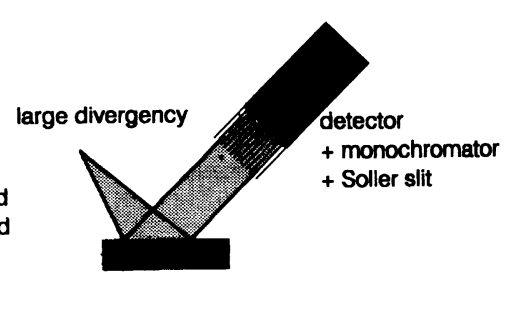

b.

FIGURE 3 Divergency slit angles (a) for the classical setup and (b) for the presented setup.

(see Fig. 3(a)). Unfortunately, the Soller slit (used for low incident beam angles) only allows for small differences in angle. The problem can be overcome by choosing a large divergency slit angle at the source. The drawback of that method is that a large area on the sample is irradiated (see Fig. 3(b)) and that slight distortions of the pole figures are possible. However, this disadvantage of the presented technique counts for little compared to the advantage of the background reduction.

\section{COMPARISON OF ODFS OBTAINED WITH DIFFERENT SETUPS}

The first example concerns a cold rolled $\mathrm{Al}$ sheet. The texture of such sample is classically measured with $\mathrm{Cu} \mathrm{K} \alpha$ radiation (see Fig. 4). A measurement with the presented setup (also $\mathrm{Cu} \mathrm{K} \alpha$ radiation, but with monochromator and Soller slit) increases the texture index from 2.85 for the classical measurement to 3.43. The ODF obtained with the presented setup is shown in Fig. 5. The explanation of the difference between the two ODFs resides in the errors in the background correction of the experimental pole figures. Such background correction has been done in both cases. This procedure requires an estimation of the background level in each pole figure. The correction is done by subtracting the estimate of the background from all pole figure intensities. There is always an error in such estimation. An error of $20 \%$ is not unusual. However, a $20 \%$ error on a low background intensity (new method) is less damaging than a $20 \%$ error on a high background intensity. 


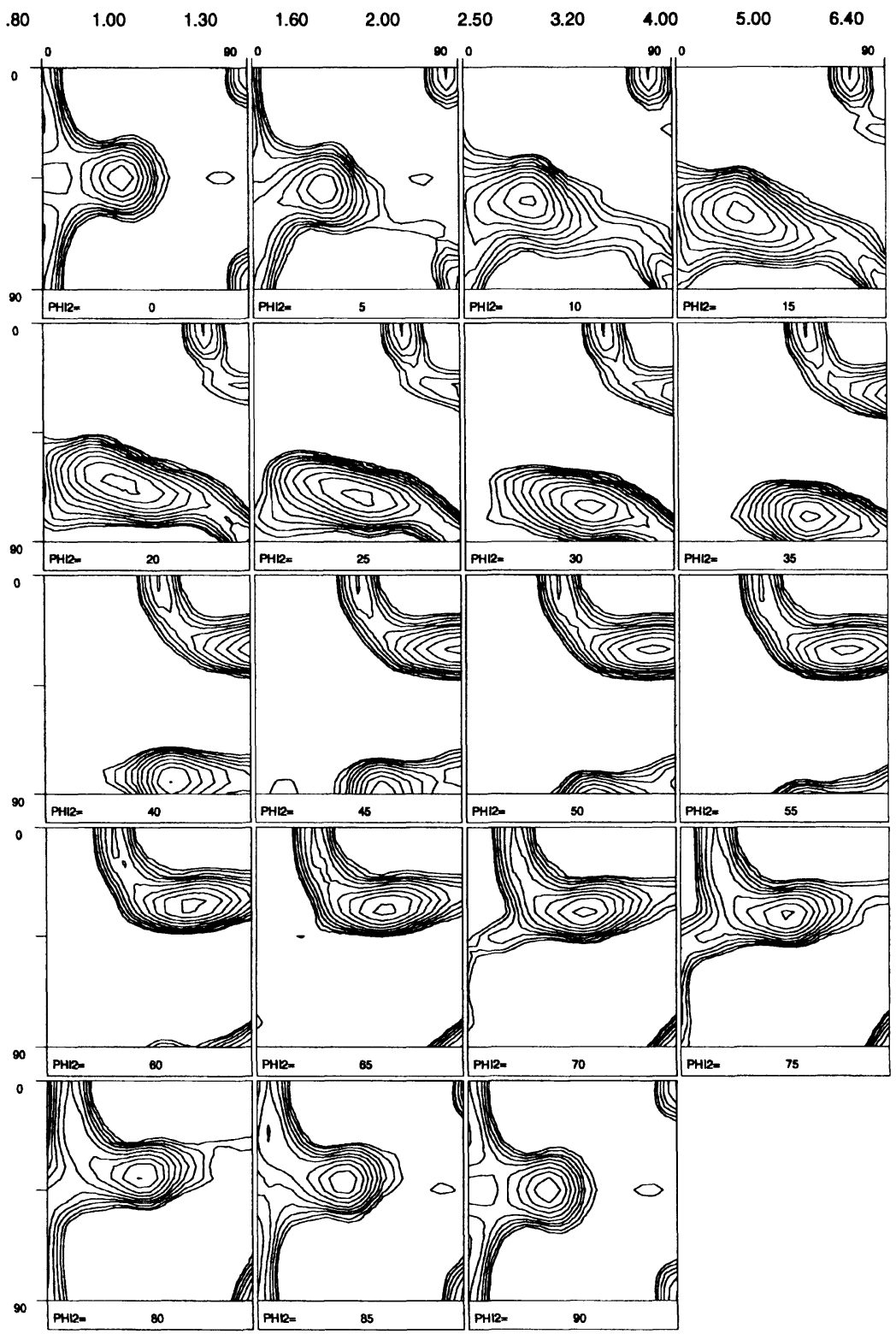

FIGURE 4 ODF of cold rolled Al sample obtained with classical setup (texture index 2.85). 


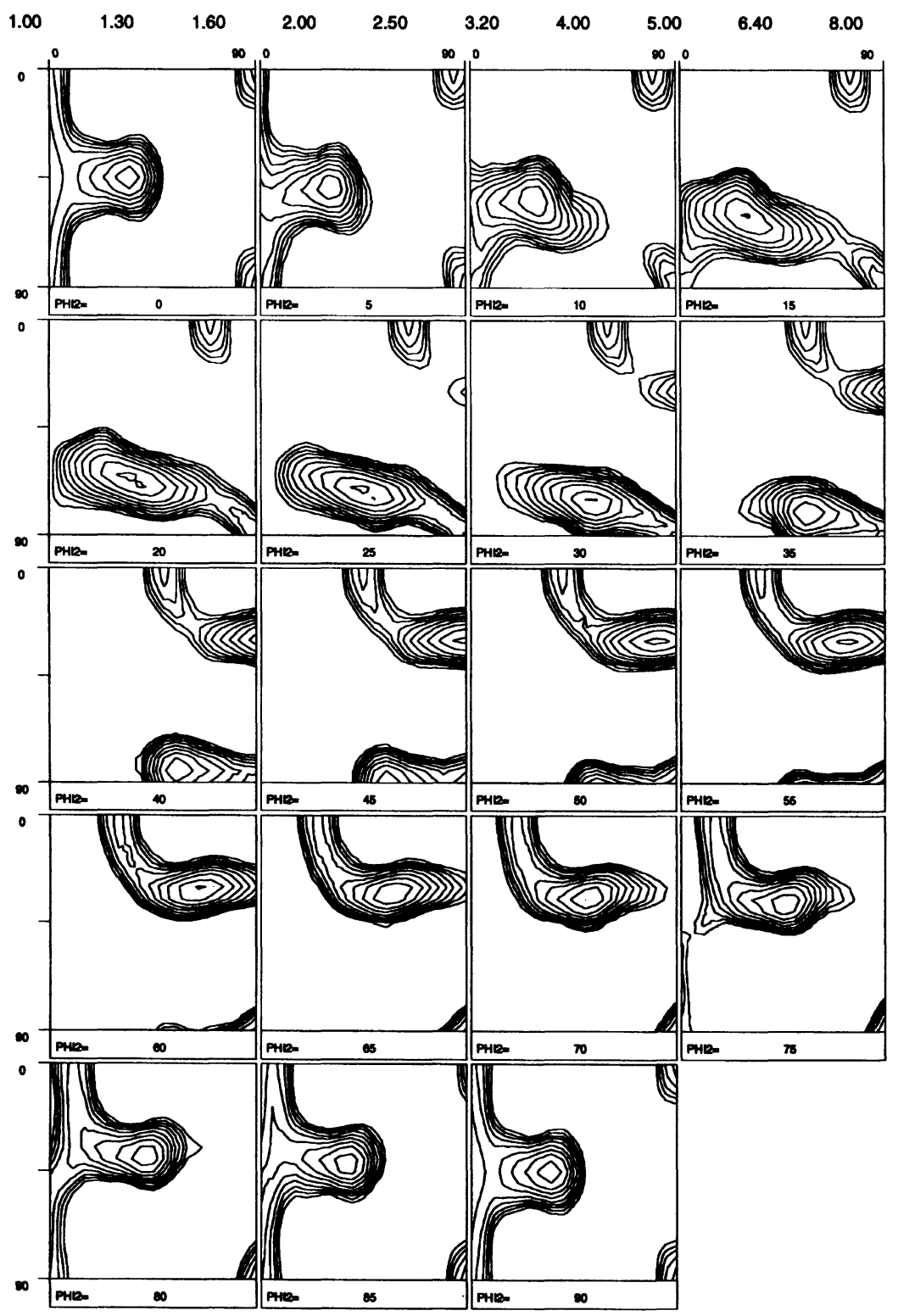

FIGURE 5 ODF of cold rolled Al sample obtained with the new setup (texture index 3.43). 
The second example concerns a cold rolled $\mathrm{Fe}$ sheet. The texture of such sample is classically measured with Mo radiation (see Fig. 6). Cu $\mathrm{K} \alpha$ is not useful for $\mathrm{Fe}$ because of the high fluorescence. However, the high background is almost cancelled by using the presented setup (with the monochromator). The ODF obtained with the presented setup is shown in Fig. 7. The texture index of the last ODF (3.42) is even higher than the texture index of the ODF obtained with Mo radiation (2.95).

\section{MONTE CARLO SIMULATION FOR THE EFFECT OF THE I/B RATIO ON THE QUALITY OF THE ODF}

Let us consider a given ODF of a certain sample. The "perfect" intensities which should be measured on a perfect instrument without background and without counting statistics can then be derived from the recalculated pole figures. These intensities are multiplied by a given factor and a given background intensity is added. Thereafter, more realistic intensities are simulated based on counting statistics (Poisson's distribution). Such simulated pole figures are then treated as if they were measured. The background correction is performed on the simulated pole figures with the (exactly known) given background intensities and the ODF is again calculated. This new ODF can be compared with the original one. The difference between new and original ODF is quantified here by the parameter sq, i.e. the sum of the squared differences of the values in the Euler grid points between original and new ODF. The lower sq, the closer the result is to the original ODF.

Figure 8 shows us that higher I/B ratios yield lower sq values. We can thus conclude that the increase of the $\mathrm{I} / \mathrm{B}$ ratio ameliorates the texture measurement, even when only counting statistics are regarded. In reality, the effect of a high background is worse when the background is badly estimated.

\section{CONCLUSIONS}

One setup consisting of a texture goniometer equipped with a Soller slit and monochromator and with a $\mathrm{Cu} \mathrm{K} \alpha$ tube could be used successfully 

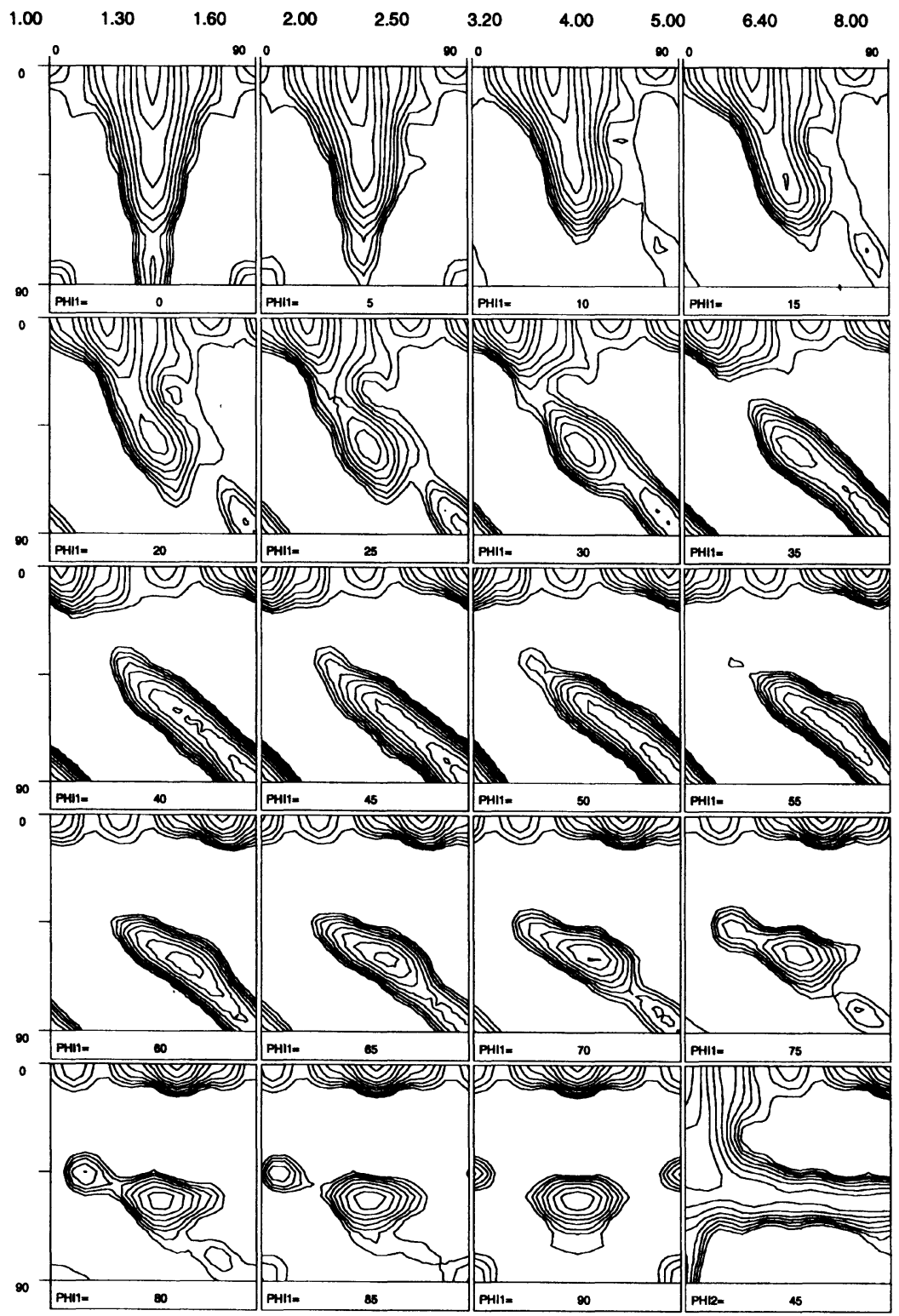

FIGURE 6 ODF of cold rolled Fe sample obtained with the Mo radiation (texture index 2.95). 

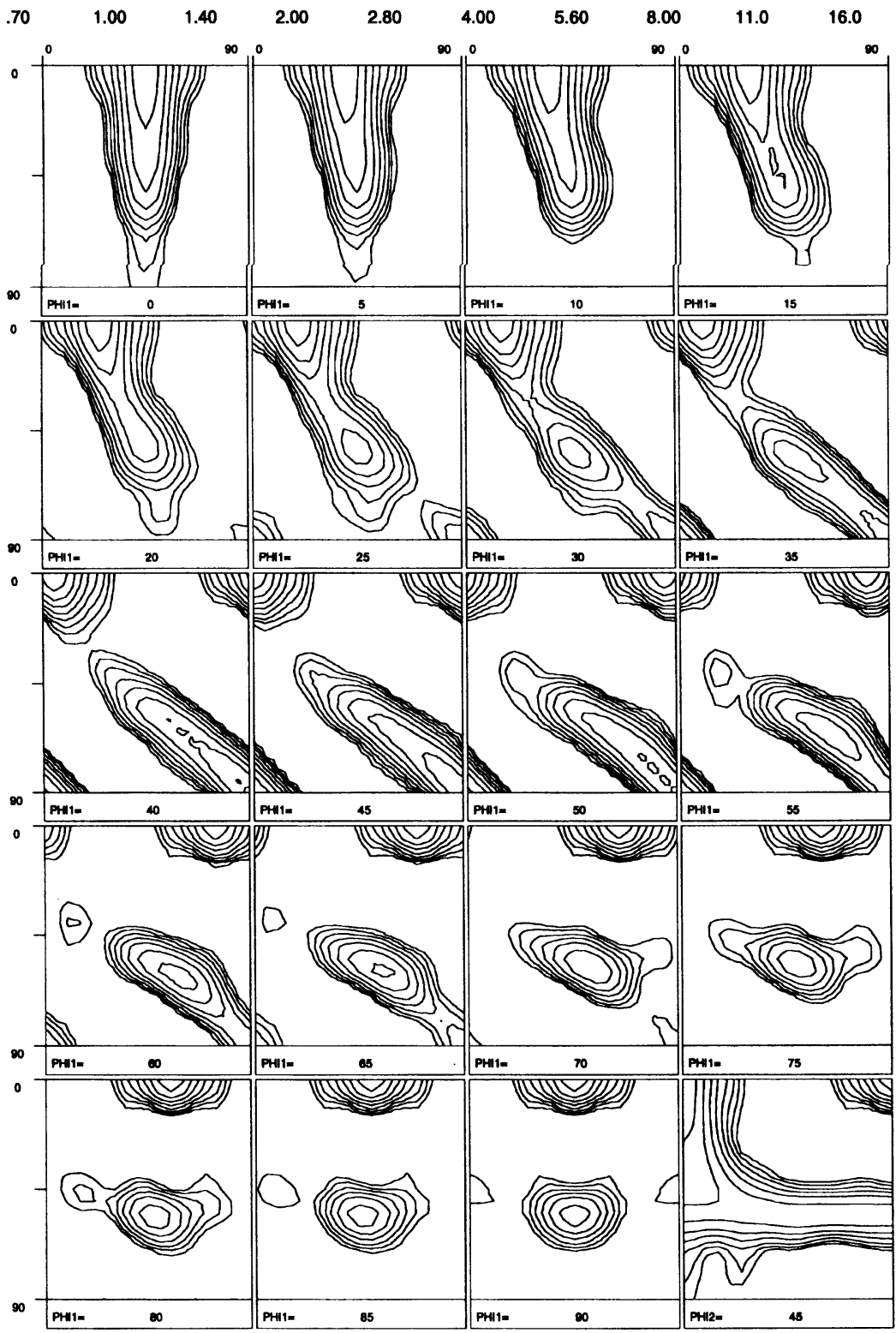

FIGURE 7 ODF of cold rolled Fe sample obtained with the new setup (texture index 3.42). 


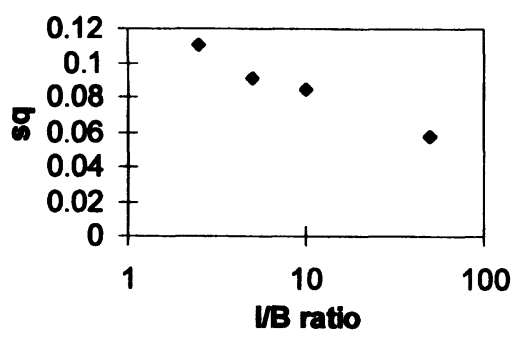

a.

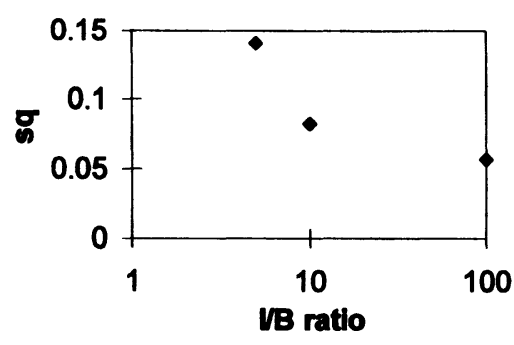

b.

FIGURE 8 sq as function of the I/B ratio (a) when the intensity is kept constant at $500 \mathrm{cps}$ while the background was varied and (b) when the background was kept constant on $10 \mathrm{cps}$ while the intensity was varied.

on $\mathrm{Al}$ and $\mathrm{Fe}$. The sharpness of the texture is higher than for the most suitable "classical" measurement, i.e. with Mo radiation on the $\mathrm{Fe}$ sample and with $\mathrm{Cu}$ radiation on the $\mathrm{Al}$. The same setup can also be used for pole figure measurements on thin coatings with a low incident beam angle. The background reduction of the monochromator, and consequently the improvement of the Intensity/Background ratio, is the strength of this method. This background reduction makes the calculated ODFs more reliable, since the calculation is less sensitive to the errors of the estimated background intensities.

\section{Acknowledgements}

This work has been made possible through the financial support through the Belgian programme on Interuniversity Poles of Attraction initiated by the Belgian State, Prime Minister's Office, Science Policy Programming (Contract IUAP-4). One of the authors (KVA) gratefully acknowledges the financial support of the N.F.W.O. (National Fund for Scientific Research, Belgium).

\section{References}

Bonarski, J.T., Wcislak, L. and Bunge, H. (1994) Investigation of inhomogeneous textures of coatings and near-surface layers. In Proceedings of 10th International Conference on the Textures of Materials (ICOTOM10), ed. Bunge, H., Mater. Sci. Forum 157-162, 111-118. 
Moreau, B., Wagner, F. and Göbel, H. (1994) Optimization of the texture determination of thin films from X-ray diffraction measurements. In Proceedings of 10th International Conference on the Textures of Materials (ICOTOM10), ed. Bunge, H., Mater. Sci. Forum 157-162, 159-166.

Schubert, A., Kampfe, B. and Auerswald, E. (1993) X-ray analysis of residual stress and textures in thin coatings. In Proceedings of 2nd European Powder Diffraction Conference (EPDIC2), eds. Delhez, R. and Mittemeijer, E., Mater. Sci. Forum 133-136, $117-122$.

Szpunar, J.A. and Blandford, P. (1994) Equipment for texture measurement in thin films. In Proceedings of 10th International Conference on the Textures of Materials (ICOTOM10), ed. Bunge, H., Mater. Sci. Forum 157-162, 207-212.

Van Acker, K., De Buyser, L., Celis, J.P. and Van Houtte, P. (1994) Characterization of thin nickel electrocoatings by means of the low incident beam angle diffraction. J. Appl. Cryst. 27, 56-66. 